\title{
Biochemical nutritional indicators in children with protein energy malnutrition attending Kanti Children Hospital, Kathmandu, Nepal
}

\author{
Mishra SK ${ }^{1}$, Bastola SP², Jha $\mathbf{B}^{3}$
}

${ }^{1}$ Assistant Professor, Department of Clinical Biochemistry, Kantipur Dental College, Kathmandu, Nepal, ${ }^{2}$ Assistant Professor, Department of Community Medicine \& Family Health, ${ }^{3}$ Professor \& Head of Department, Department of Clinical Biochemistry, Institute of Medicine, Kathmandu, Nepal

\begin{abstract}
Background: Nutrition is the science of food and its relationship to health. Nutritional status is one of the key indicators of health Nutritional status of Nepal is not satisfactory. Especially Protein Energy Malnutrition (PEM) in Nepal is a very common problem in children under five years of age. There are number biochemical parameters which become altered during protein energy malnutrition.

Objectives: The objectives of the study are to determine biochemical nutritional indicators among children suffering from PEM and to compare all biochemical parameters with well nourished children and also to determine the factors affecting PEM among children under five years.

Materials and methods: The study design was cross sectional, descriptive study with control. Children between ages 6 to 59 months attending outpatient department (OPD) and wards of Kanti Children's Hospital (KCH), Maharajgunj, Kathmandu, Nepal were purposively selected. The sample size was calculated by using prevalence of malnutrition in Nepalese context and allowable error of $10 \%$, a total of 120 cases which include 60 PEM cases and 60 controls were selected. They were grouped into two groups based on whether they are above or below the $80 \%$ of $50^{\text {th }}$ percentile of weight for height based on growth chart of National Centre of Health Statistics ${ }^{3}$ (NCHS), USA.

Results: The educational status of parents of children with PEM was found to be significantly less $(\mathrm{p}<0.05)$ as compared to their non-PEM counterparts. Occupations of parents whose children were in PEM group include mainly housewives and labourers. Larger proportions of children in our study were born at home and exact birth weights of children were not known. Most of the children are colostrum fed. Most of the children in our study were immunized. Almost equal proportion of children belonged to nuclear family type and joint family type. The mean serum glucose, sodium, potassium, cholesterol, haemoglobin was not significantly different in both groups while mean total protein, albumin, and calcium were significantly $(\mathrm{p}<0.05)$ low in PEM group when compared to well nourished children (control). There was significantly $(\mathrm{p}<0.05)$ higher incidence of hypoproteinemia, hypoalbuminaemia, and hypocalcaemia, in PEM group when compared to control group.

Conclusion: A significant proportion of children with protein energy malnutrition had altered biochemical parameters which were related to food intake and biochemical metabolism mandatory during growth and development of children less than five years of age. There was significantly higher proportion of hypoglycaemia, hypoproteinemia, hypoalbuminaemia, anaemia, hypocholesterolemia and hypocalcaemia in children with PEM when compared to normally nourished children.
\end{abstract}

Key words: PEM, Biochemical nutritional indicators, anthropometry

$\mathrm{N}$ utrition is the science of food and its relationship to health. Especially protein energy malnutrition in Nepal is a very common problem in children under five years of age. Nutritional status is one of the key indicators of health. There are number biochemical parameters which become altered during protein energy malnutrition. Protein Energy Malnutrition in early childhood is the predisposing factor that leads much of the morbidity and mortality in children under five ${ }^{1}$. There are many ways to evaluate PEM; however biochemical parameters provide the valuable information for the over-all management and act as sensitive indicators especially in a tertiary health care

\footnotetext{
Correspondence

Shravan Kumar Mishra

Assistant Professor

Department of Clinical Biochemistry

Kantipur Dental College, Kathmandu

E-mail: shravanmishra11@yahoo.com
} 
centre. Clinical and anthropometric features of PEM are late to appear. Hence biochemical parameters will prove as early and sensitive indicators of developments of PEM. Biochemical value will trace the real nutritional status and also help in optimal specific and precise management of PEM in tertiary care centre.

PEM is a range of pathological condition arising from coincident lack of protein and calories and usually associated with infections and deficiency of micronutrients. Protein Energy Malnutrition (PEM) and iodine, vitamin A and iron deficiencies increase the risk of death and disability from diarrhoea, Acute Respiratory Infection (ARI) and vaccine preventable diseases, particularly measles ${ }^{2}$. Conversely diarrhoea, parasite infestation and other child-hood ailments diminish children's ability to utilize what nutrients are available in their diets. These are conditions due to varying degrees of protein and calories deficiencies in which there is failure to maintain adequate weight gain or growth rate in the early stages but as the condition progresses, there is loss of weight associated with loss of subcutaneous fat and muscle mass. Resulting dysfunction of many vital organs leads to a variety of clinical features. With increasing severity there is increasing failure in the homeostatic mechanisms of the body and damages to the immune defences, which may result in infections and death.

\section{Materials and methods}

The study design was cross sectional, descriptive with control. Children between ages 6 to 59 months attending outpatient department (OPD) and wards of Kanti Children's Hospital were purposively selected. The study was conducted for a period of six month starting from May to October in the year 2007. The sample size was calculated by using prevalence of malnutrition in Nepalese context and allowable error of $10 \%$.There were total of 120 children enrolled in the study out of which 60 belonged to PEM group with weight for height less than $80 \%$ of $50^{\text {th }}$ percentile of the reference and 60 belonged to control group with weight for height more than $80 \%$ of $50^{\text {th }}$ percentile.

Relevant history and physical examination based on Performa of data collection sheet was completed. Height or length was taken using non stretchable steel tape and weight was taken from a weighing scale. Mid Upper Arm Circumference (MUAC) was measured using plastic tape using standard technique. After taking history and physical examination, venous blood was collected without using tourniquet and was kept in EDTA vial and test tube. The EDTA (Ethylene Diamine Tetra acetic Acid) blood was used for haemoglobin estimation by Cyanmethaemoglobin method in colorimeter. The blood sample collected in test tube was quickly centrifuged and sent to laboratory for estimation of serum biochemical parameters. Blood glucose was measured by glucose oxidase and peroxidase (GOD/POD); protein by Biuret method; albumin by bromocresol green (BCG) method; calcium by O-cresolphthalein complexone (O-CPC) method cholesterol by enzymatic end-point method in Stat Fax biochemistry analyzer. Sodium and potassium were measured by ion selective electrode (ISE) in NOVA biolyte analyzer.

The study included children between the ages of six months and fifty nine months and also those not suffering from any chronic illness and malignant diseases. The exclusion criteria of the study were children who were being given intravenous fluids and also children of parents who refused to give consent for blood examination. Data were analysed using SPSS data base. The biochemical nutritional indicators among PEM were correlated with biochemical nutritional indicators in control group. Different relevant statistical tests were done. Approval from institution was taken before carrying out study. Before drawing blood, verbal consent of parents was taken.

\section{Results}

There were total of 120 children enrolled in the study out of which 60 belonged to PEM group and 60 belonged to control group. There were 32 male and 28 female child in the study group, the same number of male and female and approximately same age were selected for controls. In both groups the ratio of male to female and also age distribution in both groups were approximately identical. The educational status of parents of children with PEM was found to be significantly less as compared to their control counterparts. Occupations of parents whose children are in PEM group include mainly housewife and labourer. Larger proportion of children in our study was born at home and exact birth weights of children are not known. Most of the children are colostrums fed. Most of the children in our study are immunised. Almost equal proportion of children belongs to nuclear family type and joint family type in both groups. The mean serum glucose, sodium, potassium, cholesterol, haemoglobin was not significantly different in both groups while mean total protein, albumin, and calcium were significantly $(p<0.05)$ low in PEM group when compared to well nourished children (control). There was significantly $(p<0.05)$ higher incidence of hypoproteinemia, hypoalbuminaemia, and hypocalcaemia, in PEM group when compared to control group.

Relative Risk of protein energy malnutrition is 3.7; this means that developing hypoproteinemia is approximately fourfold increase in risk of developing PEM when compared with control group. There was 
significant decline in total protein in the PEM group $(\mathrm{P}<0.05)$. The total protein level significantly correlated with weight for height (correlation coefficient, $\mathrm{r}=0.33$ ). This showed strong association of hypoproteinemia and protein energy malnutrition.

There was significantly higher decline in serum albumin level in PEM group as compared to control group. Relative Risk of protein energy malnutrition is 5.2; this means that developing hypoalbuminaemia is fivefold increase in risk of developing PEM when compared with control group. ).Serum albumin significantly correlated with weight for height (correlation coefficient, $\mathrm{r}=0.52$ ). This showed strong association of hypoalbuminaemia and protein energy malnutrition. Also there was no any case of hyperalbuminemia in PEM group, showing albumin as a very specific marker of protein energy malnutrition.

Relative Risk of protein energy malnutrition is 7.0; this means that developing hypocalcaemia is sevenfold increase in risk of developing PEM when compared with control group. The mean calcium in PEM group was significantly low in comparison to control group $(p<0.05)$. Serum concentration of calcium correlated significantly with weight for height (correlation coefficient, $r=0.42$ ). This showed strong association of hypocalcaemia and protein energy malnutrition.

Table 1: Serum total protein concentration in PEM and Control group

\begin{tabular}{|l|c|c|}
\hline \multicolumn{1}{|c|}{ Serum total protein } & PEM Group (\%) & Control Group (\%) \\
\hline Hypoproteinemia & $37(61.6 \%)$ & $10(16.7 \%)$ \\
\hline Normal & $22(36.7 \%)$ & $48(80.0 \%)$ \\
\hline Hyperproteinaemia & $01(1.7 \%)$ & $02(3.3 \%)$ \\
\hline Total & $60(100 \%)$ & $60(100 \%)$ \\
\hline
\end{tabular}

Table 2: Serum albumin concentration in PEM and control group

\begin{tabular}{|l|c|c|}
\hline \multicolumn{1}{|c|}{ Serum total protein } & PEM Group (\%) & Control Group (\%) \\
\hline Hypoalbuminemia & $42(70.0 \%)$ & $08(13.3 \%)$ \\
\hline Normal & $18(30.0 \%)$ & $50(83.4 \%)$ \\
\hline Hyperalbuminemia & $00(1.7 \%)$ & $02(3.3 \%)$ \\
\hline Total & $60(100 \%)$ & $60(100 \%)$ \\
\hline
\end{tabular}

Table 3: Serum calcium level in PEM and control group

\begin{tabular}{|l|c|c|}
\hline \multicolumn{1}{|c|}{ Serum Calcium } & PEM Group (\%) & Control Group (\%) \\
\hline Hypocalcaemia & $28(46.7 \%)$ & $04(6.7 \%)$ \\
\hline Normal & $32(53.3 \%)$ & $50(83.3 \%)$ \\
\hline Hypercalcaemia & $00(0.0 \%)$ & $06(10.0 \%)$ \\
\hline Total & $60(100 \%)$ & $60(100 \%)$ \\
\hline
\end{tabular}

\section{Discussion}

The educational status of parents of children with PEM was found to be significantly less as compared to their non-PEM counterparts. The result showed higher rate of illiteracy among mothers in PEM group than control group $(\mathrm{p}<0.05)$. Illiterate mothers in PEM group were twice than mothers in control group. Mothers in control group were more literate than PEM group. The result showed that education was key factor in determining the nutritional status of children especially under five. Similar types of results were obtained in two studies done in India by Aryan A, et $\mathrm{al}^{4}$ and Gupta $\mathrm{MC}$ et $\mathrm{al}^{5}$. In the study conducted by Arya A. and Devi R., the impact of maternal literacy status on the nutritional status of pre-school children was studied. Two hundred children of both the sexes aged between 1-5 years were randomly selected for the study. Results revealed that the children of literate mothers had better anthropometric measurements than children of illiterate mothers. No mothers in PEM groups were in job where as one fourth of mothers in control group are in job. Mothers in PEM group were twice in labour work than in control group. Housewife mothers, if husband is a farmer or labourer, also help their husbands in their work so have little time to care their children. PEM children were more prevalent among children whose fathers were illiterate. The education of fathers above SLC in control group was more than twice as in PEM group. The major occupations in fathers of PEM group were farmer and 
labourer and that in control group were service-holder and farmer. So the result showed children of labourer are more prone to PEM. Also fathers of control group were more than three times in job than that of PEM group. In the study conducted by Gupta MC et. $\mathrm{al}^{5}$ in India, 390 children aged 24-72 months participated. Out of these, 26 severely malnourished children weight for age 55.27 \pm 3.17 were identified in a colony of predominantly Muslim urban slum dwellers of low socio-economic status. An equal number $(n=26)$ of normally nourished children match for age, sex and per capita income were identified, a strong correlation was found between nutritional status subjects and educational level of their mothers $(p<0.025)$. Father's education was unrelated to children's nutritional status. The study showed no significant difference in the types of family structure in PEM in comparison to control group. Larger proportion of births took place at home in both groups, though more in case of PEM group. Regarding colostrum feeding there was no significant difference in both groups; however the proportion of colostrums not fed was higher in PEM group. Larger proportion children were immunized in both groups, lower immunization in case of PEM group in comparison to control group. A large proportion of births occurred at home so mothers didn't know the exact birth weight of their children. Among known cases low birth baby was higher in PEM group than control group.

The mean glucose concentration in PEM and control was not significantly different $(\mathrm{p}=0.08)$ but as the variance of serum glucose was significantly different in two groups, the prevalence of hypoglycaemia was significantly higher in PEM group in comparison to the control group however euglycaemic children were more in number than hypoglycaemic due to glucose homeostasis and rebound hyperglycaemia in hypoglycaemic condition. In spite of frequent occurrence of hypoglycaemia, the level of glucose was not extremely low, the lowest value being $41.0 \mathrm{mg} / \mathrm{dl}$. None of the cases with hypoglycaemia presented with altered consciousness showing thereby the unique capability of body to cope with low glucose. Buchanan $\mathrm{N}$ et $\mathrm{al}^{6}$ found impaired glycogenolysis in severe kwashiorkor leading to hypoglycaemia. Glyn Jones $\mathrm{R}^{7}$ got a higher incidence of hypoglycaemia, which was significantly associated with higher mortality rate. In the study conducted by Bhattarai $\mathrm{PM}^{8}$ also found similar results. A number of children of both groups were found to have hyperglycaemia but there was no significant correlation among them. According to Piyush Gupta et al $(1997)^{9}$, transient hyperglycaemia occurred as a part of stress response in acute illnesses and was brought about by elevated levels of counterregulatory hormones. Out of 758 children (1 month to 6 year) with acute illnesses 36 children (4.7\%) were found to be hyperglycaemic. In the present study the figures were $11.6 \%$ and $6.7 \%$ for PEM and non PEM groups respectively which were correlated with $13.4 \%$ and $8.4 \%$ for PEM and non PEM groups respectively in the study conducted by Bhattarai P.M. et al. ${ }^{6}$ Hyperglycemia had been noted in malnourished children with dehydrating gastroenteritis in a study conducted by Anju Seth and S. Anoja. ${ }^{10}$ Five of the fifty patients included in the study were found to be hyperglycaemia.

The mean sodium in both groups was not significantly different $(p=0.35)$. The serum sodium concentration was poorly correlated with weight for height (correlation coefficient, $r=0.12$ ). Serum sodium level was not significantly associated with nutritional status. $\mathrm{P}$ value was 0.054 for hyponatremia and 0.56 for hyponatremia. Relative Risk of protein energy malnutrition was 1.5; this means that developing hyponatremia was less than twofold increase in risk of developing PEM when compared with control group. This showed weak association of hyponatremia and protein energy malnutrition. There was no significant correlation of either hyponatremia or hyperkalaemia with nutritional status. P value for hypo- and hyponatremia are 0.052 and 0.54 respectively. Because of increase in intracellular water, the total sodium may be increased but the actual serum level may be relatively decreased as a result of excessive increase in water in comparison to increase in sodium. According to Garrow JS et $\mathrm{al}^{12}$ hyponatremia was very bad prognostics factor but according to Mittal SK et $\mathrm{al}^{13}$, serum sodium level did not seem to affect the outcome of patients. There was no significant difference in mean potassium concentration in both groups $(p=0.117)$. Serum potassium concentration was poorly correlated with weight for height (correlation coefficient, $r=0.14$ ). Serum potassium level was not significantly associated with nutritional status $\mathrm{p}$ value for hypokalaemia is 0.11 and that of hyperkalaemia is 0.55 . Relative Risk of protein energy malnutrition is 1.7 ; this meant that developing hypokalaemia was less than twofold increase in risk of developing PEM when compared with control group. This showed weak association of hypokalaemia and protein energy malnutrition. In the present study, no significant difference in serum potassium concentration was noted in PEM and control groups. This result contrasted with many other studies done in other countries. Wakwe VC et $\mathrm{al}^{14}$ found a significant decline in serum potassium level ( $p$ value $<0.001$ ) in PEM cases when compared to control group. Similar results with low serum potassium were obtained from studies conducted by Rao A et al ${ }^{15}$ and Kalra $\mathrm{K}$ et al ${ }^{16}$ in India. Occurrence of hypokalaemia could be falsely be decreased if samples taken were haemolysed as a result of improper sampling technique. In the present study all the haemolysed samples were excluded from the study. 
The difference in mean Sodium and mean Potassium in PEM and control groups was insignificant in the present study. As the whole procedure of biochemical tests were performed by investigator himself there is no possibility of interpersonal variation. Also quality control sera from Randox Company were used for quality check. Though the incidence of hypokalaemia in PEM and control group is insignificantly different, a large proportion of cases are hypokalaemia in each group; $20 \%$ in PEM group and $8.3 \%$ in control group.

The result of total protein estimation showed relative risk of protein energy malnutrition is 3.7 ; this means that developing hypoproteinemia was approximately fourfold increase in risk of developing PEM when compared with control group. This showed strong association of hypoproteinemia and protein energy malnutrition. There was significantly higher prevalence of hypoproteinemia in PEM group as compared to control group $(p<0.05)$. There was significantly higher decline in serum albumin level in PEM group as compared to control group. Relative risk of protein energy malnutrition was 5.2; this meant that developing hypoalbuminaemia was fivefold increase in risk of developing PEM when compared with control group. This showed strong association of hypoalbuminaemiaand protein energy malnutrition. Also there was no any case of hyperalbuminemia in PEM group, showing albumin as a very specific marker of protein energy malnutrition. Serum albumin significantly correlated with weight for height (correlation coefficient, $r=0.52$ ). There was significantly higher incidence of hypoalbuminaemia in PEM group as compared to control group $(p<0.05)$. The total serum protein and albumin were significantly low in PEM group in comparison to control group, $p$ value for hypoproteinemia and hypoalbuminaemia was $<0.05$. Cicely Williams had contributed the deficiency of protein as the main cause of PEM especially Kwashiorkor. There was higher degree of correlation with albumin in comparison to total protein. In multivariate analysis to analyze the prognostic value of clinical, anthropometric and biological indicators of PEM in hospitalized children serum albumin was found to be the best predictor of subsequent risk of dying. The low serum protein and albumin in PEM had also been found in studies conducted Kalra K et al (1975) ${ }^{16}$.

There was significantly higher decline in serum calcium level in PEM group as compared to control group. Relative Risk of protein energy malnutrition was 7.0; this meant that developing hypocalcaemia was sevenfold increase in risk of developing PEM when compared with control group. This showed strong association of hypocalcaemia and protein energy malnutrition. Serum concentration of calcium correlated significantly with weight for height (correlation coefficient, $r=0.42$ ). There was significantly higher incidence of hypocalcaemia in PEM group as compared to control group $(\mathrm{P}<0.05)$. In our study, the total protein and albumin were significantly low in PEM group as compared to control group so the level of calcium might have been apparently low in parallel with total protein and albumin levels. In the present study, the prevalence of hypercalcaemia was found to be higher in control group in comparison to PEM group signifying thereby the tendency of PEM cases to be either normocalcaemic or hypocalcaemic. Low serum calcium in PEM cases had also been found in study done by kalra $\mathrm{k}$ et $\mathrm{al}^{16}$ in India. In contrasts to present study and that conducted by kalra k. et al ${ }^{16}$ serum calcium (albumin adjusted) showed no significant changes in study conducted by Rao A and Cherian $\mathrm{A}^{15}$. Their study consisted of 9 cases of marasmus, 15 cases of kwashiorkor and marasmic kwashiorkor and 14 control subjects. They found no significant difference in serum sodium in PEM patients same as in present study but in contrasts to this study serum potassium was significantly lower in PEM patients.

There was significant decrease of serum cholesterol in both groups however more marked in case of PEM group. Relative Risk of protein energy malnutrition was 1.2; this means that developing hypocholesterolemia was less than twofold increase in risk of developing PEM when compared with control group. This showed weak association of hypocholesterolemia and protein energy malnutrition. There was higher prevalence of hypocholesterolemia in PEM group when compared to control group. Also higher prevalence of hypocholesterolemia was observed in control group. There was significant decrease of blood haemoglobin in both groups however more marked in case of PEM group. There was significantly higher incidence of anaemia in PEM group as compared to control group. Relative Risk of protein energy malnutrition was 2.3; this meant that developing anaemia was more than twofold increase in risk of developing PEM when compared with control group. This showed fair association of anaemia and protein energy malnutrition. There was higher prevalence of anaemia in PEM group when compared to control group. A large number of control subjects had bio-chemical abnormalities. It showed that biochemical tests were particularly helpful as early indicator of malnutrition before the actual abnormalities in anthropometric measurements can be appreciated. A limited number of samples were taken because of cost and lack of time.

\section{Conclusion}

A significant proportion of children with protein energy malnutrition had altered biochemical parameters which were related to food intake and biochemical metabolism mandatory during growth and development of children 
under five years of age. There was significantly higher proportion of hypoglycaemia, hypoproteinemia, hypoalbuminaemia, anaemia, hypocholesterolemia and hypocalcaemia in children with PEM when compared to normally nourished children. Hence the evaluation of biochemical indicators in PEM at tertiary care centre and nutritional assessment centre will be helpful for treatment and nutritional status improvement after nutritious food supplementation.

\section{References}

1. Ministry of Health and Population. Nepal Demographic \& Health Survey 2006. Kathmandu: New ERA, Macro International Inc.2007.p. 173-98.

2. UNICEF. Situation analysis on children and women in Nepal. Kathmandu: UNICEF.1996.

3. World Health Organisation. Measuring Change in Nutritional Status: Guidelines for Assessing the nutritional Impact of supplementary Feeding Programmes in Vulnerable Groups. Kathmandu: World Health Organisation. 1983.

4. Aryan A, Devi R. Influence of maternal Literacy on the nutritional status of pre-school children. Indian Journal of paediatrics 1991; 58: 265-8.

5. Gupta MC, Mehrotra M, Arora S, Saran M. Relation with childhood Malnutrition to parental education and mother's nutritional related KAP. Indian Journal of Paediatrics. 1991; 58: 269 74.

6. Buchanan N, Moodley G, Eyberg, Bloom SR, Hansen JD. Hypoglycaemia associated with severe kwashiorkor. South African Medical Journal. 1976;50(3F):1442-6.

7. Glyn Jones R. Blood sugar in infantile gastroenteritis. South African Medical Journal. 1975; 49 (36);1474-6.
8. Bhattarai PM. Biochemical parameters in protein energy malnutrition in children under five in the year. 2003 (not published when referenced).

9. Gupta P, Natarajan G, Agarwal KN. Transient Hyperglycaemia in Acute childhood illness: To attend to ignore? Indian Journal of paediatrics. 1997; 64:205-10.

10. Seth A, Aneja S. Hyperglycaemia in malnourished children with dehydrating gastroenteritis. Indian Journal of Paediatrics. 1995; 62:353-5.

11. Willis JS, Golden MH. Active and passive transport of sodium and potassium ions in erythrocytes of severely malnourished Jamaican children. European Journal of clinical nutrition. 1988; 42(8):635-45.

12. Garrow JS. The treatment and prognosis of infantile malnutrition in Jamaican Children. West Indian Medical Journal. 1962; 11:217-27.

13. Mittal SK, Saxena S, Mundkur N, Srivastava G, Gupta S. Acute diarrhoea in malnourished children: Clinical, biochemical and bacteriological profile. Indian Journal paediatrics.1980; 9(3); 247-53.

14. Wake VC, Okon KO. Plasma electrolyte pattern of children with protein energy malnutrition and children with prolonged diarrhoea. Journal of tropical paediatrics. 1995;41(1): 59-60.

15. Rao A, cherian A. Renal tubular function in PEM. Indian journal of paediatrics. 1990; 57; 405-9.

16. Kalra K, Mital VP, pal R, Goyal RK, Dayal RS. Serum electrolyte studies in malnutrition. Indian paediatrics. 1975;9(2):1135-9. 